\title{
A Variable-length scale Parameter Dependent State of Charge Estimation of Lithium Ion Batteries by Kalman Filters
}

\author{
Minkyu Kwak ${ }^{1}$, Bataa Lkhagvasuren ${ }^{1, *}$, Hong Sung Jin $^{1}$, Gyuwon Seo ${ }^{2}$, \\ Sungyool Bong ${ }^{2,3}$, Jaeyoung Lee $e^{2,3}$ \\ ${ }^{1}$ Department of mathematics, Chonnam National University, Gwangju, Republic of Korea \\ ${ }^{2}$ School of Earth Science and Environmental Engineering, Gwangju Institute of Science and \\ Technology (GIST), Gwangju, Republic of Korea \\ ${ }^{3}$ ERTL Center for Electrochemistry and Catalyst, Gwangju Institute of Science and Technology \\ (GIST), Gwangju, Republic of Korea \\ *E-mail: bataa@chonnam.ac.kr
}

Received: 4 October 2021 / Accepted: 29 November 2021 / Published: 5 January 2022

\begin{abstract}
This paper proposes a new state of charge (SOC) estimation algorithm based on Kalman filters (KF). In the first stage, the equivalent circuit model's parameters are estimated by a least square estimation window-wise, assuming a linear $S O C$ and open-circuit voltage $(O C V)$ relation. The algorithm accurately estimates the parameters and observes the changes that depend on SOC. Moreover, based on the estimated parameters, the $O C V$ values are identified. In the next stage, window-wise Kalman filter(ESKF) without hysteresis voltage and extended Kalman filter (ES-EKF) and sigma-point Kalman filter (ES-SPKF) algorithm with hysteresis voltage are executed to estimate SOC. Having fewer state equations and hysteresis parameters tuned up, the ES-EKF and ES-SPKF perform accurately and improve the results of previous algorithms. The proposed methods are validated by experiments with three different datasets obtained from lab tests. We also show SOC-OCV curve can be obtained in a simple way that replaces the time-consuming $\mathrm{C} / 30$ tests.
\end{abstract}

Keywords: Parameter estimation, State of charge, Battery, Kalman filter, Battery management system

\section{FULL TEXT}

(C) 2022 The Authors. Published by ESG (www.electrochemsci.org). This article is an open access article distributed under the terms and conditions of the Creative Commons Attribution license (http://creativecommons.org/licenses/by/4.0/). 